\title{
Clinical internship at the Department of Dermatology, Venereology and Allergology, Medical University of Gdansk
}

Assistants from the Department of Dermatovenerology, Grodno State Medical University, Alena Yarmolik, MD, PhD, and Nastassia Brynina, MD, participated in a clinical internship at the Department of Dermatology, Venereology and Allergology, Medical University of Gdansk, from July $3^{\text {rd }}$ to July $14^{\text {th }}$, 2017. The assistants had the opportunity to become acquainted with the work of the department, learn the rules of qualifying patients for hospitalization and participate in the process of diagnosis and treatment planning of newly admitted patients. The doctors from Grodno participated also in clinical meetings, where they discussed the latest reports from Polish and foreign scientific conferences and reviewed the current dermatological publications. In this way, the dermatologists from Grodno and Gdansk had an opportunity to share their clinical experience and present the most interesting clinical cases. The guests from Grodno were particularly interested in the work of the Dermatological Surgery ward. The assistants actively participated in diagnostic and dermatosurgical procedures in patients with skin tumors. Moreover, they gained new experience during a videodermoscopy workshop (Figure 1).

The training program also involved work at the Dermatological Outpatient Clinic and Mycological Laboratory. The assistants participated in the consultation of patients referred to the outpatient clinic due to mastocytosis of the skin - a relatively rare skin dermatosis. While working in the Outpatient Clinic of Allergic Skin Diseases, the guests from Grodno were trained in allergic diagnostic procedures, including both prick and patch tests as well as the interpretation of the test results. Due to the fact that the Dermatology Clinic in Gdansk is the only centre in the Northern Poland dealing with skin diseases in children, the assistants had a chance to become acquainted with the treatment of dermatoses in pediatric patients. The course comprised also a series of lectures on atopic dermatitis, bacterial skin diseases, psoriasis, vasculitis and sexually transmitted diseases.

In the opinion of the guests from Grodno, although the training program lasted only 2 weeks, given the in-

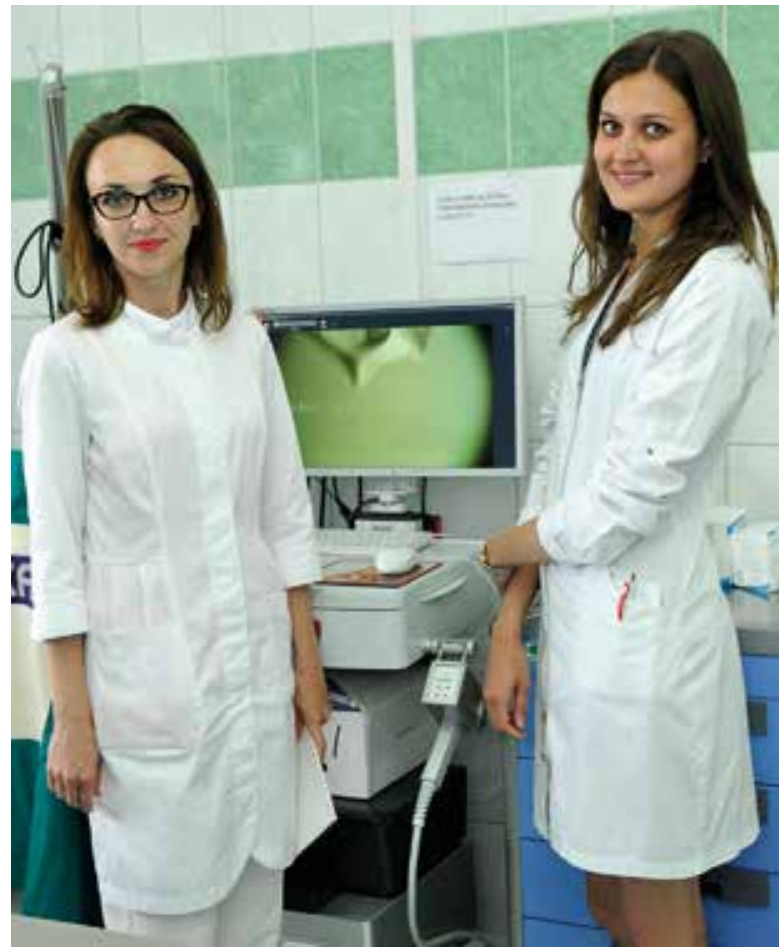

Figure 1. Assistants from the Department of Dermatovenerology in Grodno during a videodermoscopy workshop

tensity of the work and the lectures, it was sufficient for them to become well acquainted with the dermatological school of Gdansk and to gain new experience and skills. The head of the Clinic, Professor Roman J. Nowicki, handed the certificates of training to Dr. Alena Yarmolik and Dr. Nastassia Brynina and invited to continue the Polish Belarusian dermatological cooperation.

Dorota Purzycka-Bohdan, Roman J. Nowicki Department of Dermatology, Venereology and Allergology Medical University of Gdansk 